\title{
Revisiting the multifractal analysis of measures
}

\author{
Fathi Ben Nasr and Jacques Peyrière
}

\begin{abstract}
New proofs of theorems on the multifractal formalism are given. They yield results even at points $q$ for which Olsen's functions $b(q)$ and $B(q)$ differ. Indeed, we provide an example of a measure for which the functions $b$ and $B$ differ and for which the Hausdorff dimensions of the sets $X_{\alpha}$ (the level sets of the local Hölder exponent) are given by the Legendre transform of $b$ and their packing dimensions by the Legendre transform of $B$.
\end{abstract}

\section{Introduction}

The multifractal formalism aims at expressing the dimension of the level sets of the local Hölder exponent of some set function $\mu$ in terms of the Legendre transform of some "free energy" function (see [7], [5], and [6] for early works). If such a formula holds, one says that $\mu$ satisfies the multifractal formalism. At first, the formalism used "boxes", or in other terms took place in a totally disconnected metric space. In this context, the closeness to large deviation theory is patent. To get rid of these boxes and have a formalism meaningful in geometric measure theory, Olsen [8] introduced a formalism which is now commonly used. See also Pesin's monograph [9] on multifractality and dynamical systems. At this stage of the theory, whether it dealt with boxes or not, the formalism was proven to hold when there exists an auxiliary measure, a so-called Gibbs measure. Later, it was shown that this formalism holds under the condition that Olsen's Hausdorff-like multifractal measure be positive (see [2] in the totally disconnected case, [3] in general). So, the situation when $b(q)=B(q)$ (in Olsen's notation) is fairly well understood.

Here, we elaborate on the previous proofs. There is a vector version of Olsen's constructions [10], and, in particular, of the functions $b$ and $B$. However, in this setting $b$ and $B$ are functions of several variables. In this work, we show that the restriction of these functions to a suitable affine subspace can be used to estimate the Hausdorff and Tricot dimensions of some level sets. In particular, this gives

Mathematics Subject Classification (2010): 28A80, 28A78, 28A12, 11K55.

Keywords: Hausdorff dimension, packing dimension, fractal, multifractal. 
some results even in the case when $b \neq B$. Although notation is inherently complicated, we provide a simple proof of already known results, and we obtain some new estimates. In particular, we provide an example of a measure on the interval $[0,1]$ for which the functions $b$ and $B$ differ and for which the Hausdorff dimensions of the sets $X_{\alpha}$ (the level sets of the local Hölder exponent) are given by the Legendre transform of $b$, and their packing dimensions by the Legendre transform of $B$.

\section{Notations and definitions}

We deal with a metric space $(\mathbb{X}, \mathrm{d})$ having the Besicovitch property:

There exists an integer constant $C_{B}$ such that one can extract $C_{B}$ countable families $\left\{\left\{\mathrm{B}_{j, k}\right\}_{k}\right\}_{1 \leq j \leq C_{B}}$ from any collection $\mathcal{B}$ of balls so that

1. $\bigcup_{j, k} \mathrm{~B}_{j, k}$ contains the centers of the elements of $\mathcal{B}$,

2. for any $j$ and $k \neq k^{\prime}, \mathrm{B}_{j, k} \cap \mathrm{B}_{j, k^{\prime}}=\emptyset$.

\section{Notations}

$\mathrm{B}(x, r)$ stands for the open ball $\mathrm{B}(x, r)=\{y \in \mathbb{X} ; \mathrm{d}(x, y)<r\}$. The letter $\mathrm{B}$ with or without a subscript will implicitly stand for such a ball. When dealing with a collection of balls $\left\{\mathrm{B}_{i}\right\}_{i \in I}$, the notation $\mathrm{B}_{i}=\mathrm{B}\left(x_{i}, r_{i}\right)$ will implicitly be assumed.

By a $\delta$-cover of $E \subset \mathbb{X}$, we mean a collection of balls of radii not exceeding $\delta$ whose union contains $E$. A centered cover of $E$ is a cover of $E$ consisting of balls whose centers belong to $E$.

By a $\delta$-packing of $E \subset \mathbb{X}$, we mean a collection of disjoint balls of radii not exceeding $\delta$ centered in $E$.

By a Besicovitch $\delta$-cover of $E \subset \mathbb{X}$, we mean a centered $\delta$-cover of $E$ which can be decomposed into $C_{B}$ packings.

If $E$ is a subset of $\mathbb{X}, \operatorname{dim}_{H} E$ stands for its Hausdorff $\operatorname{dimension}$ and $\operatorname{dim}_{P} E$ for its packing dimension (introduced by Tricot [12]).

Let $\mathscr{B}$ stand for the set of balls of $\mathbb{X}$ and $\mathscr{F}$ for the set of maps from $\mathscr{B}$ to $[0,+\infty)$.

The set of $\mu \in \mathscr{F}$ such that $\mu(\mathrm{B})=0$ implies $\mu\left(\mathrm{B}^{\prime}\right)=0$ for all $\mathrm{B}^{\prime} \subset \mathrm{B}$ will be denoted by $\mathscr{F}^{*}$. For such a $\mu$, one defines its support $\mathrm{S}_{\mu}$ to be the complement of the set

$$
\bigcup\{\mathrm{B} \in \mathscr{B} ; \mu(\mathrm{B})=0\} .
$$

\section{Multifractal measures and separator functions}

For $\mu=\left(\mu_{1}, \ldots, \mu_{m}\right) \in \mathscr{F}^{m}, E \subset \mathbb{X}, q=\left(q_{1}, \ldots, q_{m}\right) \in \mathbb{R}^{m}, t \in \mathbb{R}$, and $\delta>0$, one sets

$$
\overline{\mathscr{P}}_{\mu, \delta}^{q, t}(E)=\sup \left\{\sum^{*} r_{j}^{t} \prod_{k=1}^{m} \mu_{k}\left(\mathrm{~B}_{j}\right)^{q_{k}} ;\left\{\mathrm{B}_{j}\right\} \text { a } \delta \text {-packing of } E\right\},
$$


where $*$ means that one only sums the terms for which $\prod_{k} \mu_{k}\left(B_{j}\right) \neq 0$,

$$
\begin{aligned}
& \overline{\mathscr{P}}_{\mu}^{q, t}(E)=\lim _{\delta \searrow 0} \overline{\mathscr{P}}_{\mu, \delta}^{q, t}(E), \\
& \mathscr{P}_{\mu}^{q, t}(E)=\inf \left\{\sum \overline{\mathscr{P}}_{\mu}^{q, t}\left(E_{j}\right) ; E \subset \bigcup E_{j}\right\},
\end{aligned}
$$

and

$$
\begin{aligned}
& \overline{\mathscr{H}}_{\mu, \delta}^{q, t}(E)=\inf \left\{\sum^{*} r_{j}^{t} \prod_{k=1}^{m} \mu_{k}\left(\mathrm{~B}_{j}\right)^{q_{k}} ;\left\{\mathrm{B}_{j}\right\} \text { a centered } \delta \text {-cover of } E\right\}, \\
& \overline{\mathscr{H}}_{\mu}^{q, t}(E)=\lim _{\delta \searrow 0} \overline{\mathscr{H}}_{\mu, \delta}^{q, t}(E), \\
& \mathscr{H}_{\mu}^{q, t}(E)=\sup \left\{\overline{\mathscr{H}}_{\mu}^{q, t}(F) ; F \subset E\right\},
\end{aligned}
$$

It is known that $\overline{\mathscr{H}}_{\mu}^{q, t}$ is $\sigma$-subadditive, and that $\mathscr{P}_{\mu}^{q, t}$ and $\mathscr{H}_{\mu}^{q, t}$ are outer measures. When $\mathrm{d}$ is an ultrametric, then $\mathscr{H}_{\mu}^{q, t}=\overline{\mathscr{H}}_{\mu}^{q, t}$.

When $m=1$, these measures have been defined by Olsen [8]. When $\mu$ is identically 1 these quantities do not depend on $q$. They will be simply denoted by $\overline{\mathscr{P}}_{\delta}^{t}(E), \overline{\mathscr{P}}^{t}(E), \mathscr{P}^{t}(E), \overline{\mathscr{H}}_{\delta}^{t}(E), \overline{\mathscr{H}}^{t}(E)$, and $\mathscr{H}^{t}(E)$, respectively. They are the classical packing pre-measures and measures introduced by Tricot [12], and the Hausdorff centered pre-measures and measures [11]. The centered Hausdorff measures also define the Hausdorff dimension.

It will prove convenient to use the following notations, when $m=1$ :

$$
\bar{\mu}_{\delta}=\overline{\mathscr{H}}_{\mu, \delta}^{1,0}, \quad \bar{\mu}=\overline{\mathscr{H}}_{\mu}^{1,0}, \quad \text { and } \quad \mu^{\sharp}=\mathscr{H}_{\mu}^{1,0} .
$$

Also, as usual, one considers the following functions:

$$
\begin{aligned}
\tau_{\mu, E}(q) & =\inf \left\{t \in \mathbb{R} ; \overline{\mathscr{P}}_{\mu}^{q, t}(E)=0\right\}=\sup \left\{t \in \mathbb{R} ; \overline{\mathscr{P}}_{\mu}^{q, t}(E)=\infty\right\} \\
B_{\mu, E}(q) & =\inf \left\{t \in \mathbb{R} ; \mathscr{P}_{\mu}^{q, t}(E)=0\right\}=\sup \left\{t \in \mathbb{R} ; \mathscr{P}_{\mu}^{q, t}(E)=\infty\right\}, \\
b_{\mu, E}(q) & =\inf \left\{t \in \mathbb{R} ; \mathscr{H}_{\mu}^{q, t}(E)=0\right\}=\sup \left\{t \in \mathbb{R} ; \mathscr{H}_{\mu}^{q, t}(E)=\infty\right\} .
\end{aligned}
$$

It is well known [8], [10] that $\tau$ and $B$ are convex and that $b \leq B \leq \tau$. Let $J_{\tau}, J_{B}$, and $J_{b}$ stand for the interiors of the sets where respectively $\tau, B$, and $b$ are finite.

When $\mu$ is identically 1 we will denote these quantities by $\overline{\operatorname{dim}}_{B} E$, $\operatorname{dim}_{P} E$, and $\operatorname{dim}_{H} E$. The first one is the Minkowski-Bouligand dimension (or upper boxdimension), the second is the Tricot (packing) dimension [12], and the last the Hausdorff dimension.

Here is an alternate definition of $\tau_{\mu, E}$. Fix $\lambda<1$ and define

$$
\begin{aligned}
& \widetilde{\mathscr{P}}_{\mu, \delta}^{q, t}(E)=\sup \left\{\sum^{*} r_{j}^{t} \prod_{k=1}^{m} \mu_{k}\left(\mathrm{~B}_{j}\right)^{q_{k}} ;\left\{\mathrm{B}_{j}\right\} \text { a packing of } E \text { with } \lambda \delta<r_{j} \leq \delta\right\}, \\
& \widetilde{\mathscr{P}}_{\mu}^{q, t}(E)=\varlimsup_{\delta \searrow 0} \widetilde{\mathscr{P}}_{\mu, \delta}^{q, t}(E), \\
& \widetilde{\tau}_{\mu, E}(q)=\sup \left\{t \in \mathbb{R} ; \widetilde{\mathscr{P}}_{\mu}^{q, t}(E)=+\infty\right\} .
\end{aligned}
$$


Lemma 2.1. One has $\widetilde{\tau}_{\mu, E}=\tau_{\mu, E}$.

Proof. Obviously $\widetilde{\mathscr{P}}_{\mu}^{q, t}(E) \leq \overline{\mathscr{P}}_{\mu}^{q, t}(E)$, so $\widetilde{\tau}_{\mu, E} \leq \tau_{\mu, E}$. To prove the converse inequality, one only has to consider the case $\tau_{\mu, E}(q)>-\infty$.

Choose $\gamma<\tau_{\mu, E}(q)$ and $\varepsilon>0$ such that $\gamma+\varepsilon<\tau_{\mu, E}(q)$. There exists $n_{0}$ such that, for all $n>n_{0}$, there exists a $\lambda^{n}$-packing $\left\{\mathrm{B}_{j}\right\}$ of $E$ such that

$$
\sum r_{j}^{\gamma+\varepsilon} \prod_{k=1}^{m} \mu_{k}\left(\mathrm{~B}_{j}\right)^{q_{k}}>1
$$

As

$$
\sum r_{j}^{\gamma+\varepsilon} \prod_{k=1}^{m} \mu_{k}\left(\mathrm{~B}_{j}\right)^{q_{k}}=\sum_{i \geq 0} \sum_{\lambda<r_{j} \lambda^{-(n+i)} \leq 1} r_{j}^{\gamma+\varepsilon} \prod_{k=1}^{m} \mu_{k}\left(\mathrm{~B}_{j}\right)^{q_{k}},
$$

there exists $i \geq 0$ such that

$$
\sum_{\lambda<r_{j} \lambda^{-(n+i)} \leq 1} r_{j}^{\gamma+\varepsilon} \prod_{k=1}^{m} \mu_{k}\left(\mathrm{~B}_{j}\right)^{q_{k}}>\lambda^{i \varepsilon}\left(1-\lambda^{\varepsilon}\right),
$$

from which it follows

$$
\sum_{\lambda<r_{j} \lambda^{-(n+i)} \leq 1} r_{j}^{\gamma} \prod_{k=1}^{m} \mu_{k}\left(\mathrm{~B}_{j}\right)^{q_{k}}>\lambda^{-(n+i) \varepsilon} \lambda^{i \varepsilon}\left(1-\lambda^{\varepsilon}\right)=\lambda^{-n}\left(1-\lambda^{\varepsilon}\right),
$$

and $\widetilde{\mathscr{P}}_{\mu}^{q, \gamma}(E)=+\infty$.

Corollary 2.2. For any $\lambda<1$, one has

$$
\begin{aligned}
\tau_{\mu, E}(q)=\varlimsup_{\delta \searrow 0} \frac{-1}{\log \delta} \log \sup \left\{\sum_{k=1}^{*}\right. & \prod_{k}^{m} \mu_{k}\left(\mathrm{~B}_{j}\right)^{q_{k}} ; \\
& \left.\left\{\mathrm{B}_{j}\right\} \text { a packing of } E \text { with } \lambda \delta<r_{j} \leq \delta\right\} .
\end{aligned}
$$

\section{Level sets of local Hölder exponents}

Let $\mu$ be an element of $\mathscr{F}^{*}$. For $\alpha, \beta \in \mathbb{R}$, one sets

$$
\begin{aligned}
\bar{X}_{\mu}(\alpha) & =\left\{x \in \mathrm{S}_{\mu} ; \varlimsup_{r \searrow 0} \frac{\log \mu(\mathrm{B}(x, r))}{\log r} \leq \alpha\right\}, \\
\underline{X}_{\mu}(\alpha) & =\left\{x \in \mathrm{S}_{\mu} ; \frac{\lim }{r \searrow 0} \frac{\log \mu(\mathrm{B}(x, r))}{\log r} \geq \alpha\right\}, \\
X_{\mu}(\alpha, \beta) & =\underline{X}_{\mu}(\alpha) \cap \bar{X}_{\mu}(\beta),
\end{aligned}
$$

and

$$
X_{\mu}(\alpha)=\underline{X}_{\mu}(\alpha) \cap \bar{X}_{\mu}(\alpha)
$$




\section{Results}

First, we revisit the Billingsley and Tricot lemmas [4], [12].

Lemma 3.1. Let $E$ be a subset of $\mathbb{X}$ and $\nu$ an element of $\mathscr{F}$.

a) If $B_{\nu, E}(1) \leq 0$, then

$$
\begin{aligned}
& \operatorname{dim}_{H} E \leq \sup _{x \in E} \frac{\lim }{r \searrow 0} \frac{\log \nu(\mathrm{B}(x, r))}{\log r}, \\
& \operatorname{dim}_{P} E \leq \sup _{x \in E} \varlimsup_{r \searrow 0} \frac{\log \nu(\mathrm{B}(x, r))}{\log r}
\end{aligned}
$$

b) If $\nu^{\sharp}(E)>0$, then

$$
\begin{aligned}
& \operatorname{dim}_{H} E \geq \operatorname{esssup}_{x \in E, \nu^{\sharp}} \frac{\lim }{r \searrow 0} \frac{\log \nu(\mathrm{B}(x, r))}{\log r}, \\
& \operatorname{dim}_{P} E \geq \operatorname{esssup}_{x \in E, \nu \sharp} \varlimsup_{r \searrow 0} \frac{\log \nu(\mathrm{B}(x, r))}{\log r},
\end{aligned}
$$

where

$$
\operatorname{esssup}_{x \in E, \nu^{\sharp}} \chi(x)=\inf \left\{t \in \mathbb{R} ; \nu^{\sharp}(E \cap\{\chi>t\})=0\right\} .
$$

Proof. Take

$$
\gamma>\sup _{x \in E} \frac{\lim }{r \searrow 0} \frac{\log \nu(\mathrm{B}(x, r))}{\log r}
$$

and $\eta>0$. Since $B_{\nu, E}(1) \leq 0$ there exists a partition $E=\bigcup E_{j}$ such that $\sum \overline{\mathscr{P}}_{\nu}^{1, \eta / 2}\left(E_{j}\right)<1$. Therefore we have that $\sum \overline{\mathscr{P}}_{\nu}^{1, \eta}\left(E_{j}\right)=0$.

Let $F$ be a subset of $E_{k}$ and let $\delta$ be a positive number. For all $x \in F$, there exists $r \leq \delta$ such that $\nu(\mathrm{B}(x, r)) \geq r^{\gamma}$. By the Besicovitch property, there exists a centered $\delta$-cover $\left\{\mathrm{B}_{j}\right\}$ of $F$, which can be decomposed into $C_{B}$ packings, such that $\nu\left(\mathrm{B}_{j}\right) \geq r_{j}^{\gamma}$. We then have

$$
\sum r_{j}^{\gamma+\eta} \leq \sum r_{j}^{\eta} \nu\left(\mathrm{B}_{j}\right) \leq C_{B} \overline{\mathscr{P}}_{\nu, \delta}^{1, \eta}\left(E_{k}\right)
$$

Therefore we have $\overline{\mathscr{H}}^{\gamma+\eta}(F)=0, \mathscr{H}^{\gamma+\eta}\left(E_{k}\right)=0$, and finally $\mathscr{H}^{\gamma+\eta}(E)=0$. Then (3.1) easily follows.

To prove (3.2), take

$$
\gamma>\sup _{x \in E} \varlimsup_{r \searrow 0} \frac{\log \nu(\mathrm{B}(x, r))}{\log r}
$$

and $\eta>0$. As previously, there exists a partition $E=\bigcup E_{j}$ such that $\sum \overline{\mathscr{P}}_{\nu}^{1, \eta}\left(E_{j}\right)=0$.

For all $x \in E$, there exists $\delta>0$ such that, for all $r \leq \delta$, one has $\nu(\mathrm{B}(x, r)) \geq r^{\gamma}$. Consider the set

$$
E(n)=\left\{x \in E ; \forall r \leq 1 / n, \nu(\mathrm{B}(x, r)) \geq r^{\gamma}\right\} .
$$


Let $\left\{\mathrm{B}_{j}\right\}$ be a $\delta$-packing of $E_{k} \cap E(n)$, with $\delta \leq 1 / n$. One has

$$
\sum r_{j}^{\gamma+\eta} \leq \sum_{j} r_{j}^{\eta} \nu\left(\mathrm{B}_{j}\right) \leq \overline{\mathscr{P}}_{\nu, \delta}^{1, \eta}\left(E_{k}\right)
$$

from which $\overline{\mathscr{P}}^{\gamma+\eta}\left(E_{k} \cap E(n)\right)=0$ follows.

So we have $\mathscr{P}^{\gamma+\eta}(E(n))=0$. Since $E=\bigcup_{n \geq 1} E(n)$, one has $\operatorname{dim}_{P} E \leq \gamma+\eta$, and hence (3.2).

To prove (3.3), take

$$
\gamma<\operatorname{ess}_{x \in E, \nu \sharp} \frac{\lim }{r \searrow 0} \frac{\log \nu(\mathrm{B}(x, r))}{\log r}
$$

and consider the set $F=\left\{x \in E ; \underline{\lim }_{r \searrow 0} \frac{\log \nu(\mathrm{B}(x, r))}{\log r}>\gamma\right\}$. We have $\nu^{\sharp}(F)>0$. For all $x \in F$, there exists $\delta>0$ such that, for all $r \leq \delta$, one has $\nu(\mathrm{B}(x, r)) \leq r^{\gamma}$. Consider the set

$$
F(n)=\left\{x \in F ; \forall r \leq 1 / n, \nu(\mathrm{B}(x, r)) \leq r^{\gamma}\right\}
$$

We have $F=\bigcup_{n \geq 1} F(n)$. Since $\nu^{\sharp}(F)>0$, there exists $n$ such that $\nu^{\sharp}(F(n))>0$, and therefore there is a subset $G$ of $F(n)$ such that $\bar{\nu}(G)>0$. Then for any centered $\delta$-cover $\left\{\mathrm{B}_{j}\right\}$ of $G$, with $\delta \leq 1 / n$, one has

$$
\bar{\nu}_{\delta}(G) \leq \sum \nu\left(\mathrm{B}_{j}\right) \leq \sum r_{j}^{\gamma} .
$$

Therefore,

$$
\bar{\nu}_{\delta}(G) \leq \overline{\mathscr{H}}_{\delta}^{\gamma}(G), \quad \text { and } \quad 0<\bar{\nu}(G) \leq \overline{\mathscr{H}}^{\gamma}(G) \leq \mathscr{H}^{\gamma}(G),
$$

which implies $\operatorname{dim}_{H} E \geq \operatorname{dim}_{H} G \geq \gamma$.

To prove (3.4), take

$$
\gamma<\operatorname{ess}_{x \in E, \nu \sharp} \varlimsup_{r \searrow 0} \frac{\log \nu(\mathrm{B}(x, r))}{\log r}
$$

and consider the set $F=\left\{x \in E ; \varlimsup_{\lim _{r} \searrow 0} \frac{\log \nu(\mathrm{B}(x, r))}{\log r}>\gamma\right\}$. We have $\nu^{\sharp}(F)>0$, so there exists a subset $F^{\prime}$ of $F$ such that $\bar{\nu}\left(F^{\prime}\right)>0$. Let $G$ be a subset of $F^{\prime}$. Then, for all $x \in G$, for all $\delta>0$, there exists $r \leq \delta$ such that $\nu(\mathrm{B}(x, r)) \leq r^{\gamma}$. Then for all $\delta$, by using the Besicovitch property, there exists a collection $\left\{\left\{\mathrm{B}_{j, k}\right\}_{j}\right\}_{1 \leq k \leq C_{B}}$ of $\delta$-packings of $G$ which together cover $G$ and such that $\nu\left(\mathrm{B}_{j, k}\right) \leq r_{j, k}^{\gamma}$. Then one has

$$
\bar{\nu}_{\delta}(G) \leq \sum_{j, k} \nu\left(\mathrm{B}_{j, k}\right) \leq \sum r_{j, k}^{\gamma}
$$


This implies that there exists $k$ such that $\sum_{j} r_{j, k}^{\gamma} \geq \frac{1}{C_{B}} \bar{\nu}_{\delta}(G)$. So we have $\overline{\mathscr{P}}_{\delta}^{\gamma}(G) \geq \frac{1}{C_{B}} \bar{\nu}_{\delta}(G)$. This implies $\overline{\mathscr{P}}^{\gamma}(G) \geq \frac{1}{C_{B}} \bar{\nu}(G)$. Hence, if $F^{\prime}=\bigcup G_{j}$, one has

$$
\sum \overline{\mathscr{P}}^{\gamma}\left(G_{j}\right) \geq \frac{1}{C_{B}} \sum \bar{\nu}\left(G_{j}\right) \geq \frac{1}{C_{B}} \bar{\nu}\left(F^{\prime}\right)>0
$$

so $\mathscr{P}^{\gamma}\left(F^{\prime}\right)>0$. Therefore, $\operatorname{dim}_{P} F \geq \gamma$. Then (3.4) easily follows.

Lemma 3.2. Let $\mu$ and $\nu$ be elements of $\mathscr{F}^{*}$ and $\mathscr{F}$ respectively. Set $\varphi(t)=$ $B_{(\mu, \nu), \mathrm{S}_{\mu}}(t, 1)$ and assume that $\varphi(0)=0$ and $\nu^{\sharp}\left(\mathrm{S}_{\mu}\right)>0$. Then one has

$$
\nu^{\sharp}\left({ }^{\mathrm{C}} X_{\mu}\left(-\varphi_{r}^{\prime}(0),-\varphi_{l}^{\prime}(0)\right)\right)=0,
$$

where $\varphi_{l}^{\prime}$ and $\varphi_{r}^{\prime}$ are the left-hand and right-hand derivatives of $\varphi$.

The same result holds with $\varphi(t)=\tau_{(\mu, \nu), \mathrm{S}_{\mu}}(t, 1)$.

Proof. Take $\gamma>-\varphi_{l}^{\prime}(0)$, and choose $\gamma^{\prime}$ and $t>0$ such that $\gamma>\gamma^{\prime}>-\varphi_{l}^{\prime}(0)$ and $\varphi(-t)<\gamma^{\prime} t$. Then $\mathscr{P}_{(\mu, \nu)}^{(-t, 1), \gamma^{\prime} t}\left(\mathrm{~S}_{\mu}\right)=0$, so there exists a countable partition $\mathrm{S}_{\mu}=\bigcup E_{j}$ of $\mathrm{S}_{\mu}$ such that

$$
\sum_{j} \overline{\mathscr{P}}_{(\mu, \nu)}^{(-t, 1), \gamma^{\prime} t}\left(E_{j}\right) \leq 1
$$

and therefore $\overline{\mathscr{P}}_{(\mu, \nu)}^{(-t, 1), \gamma t}\left(E_{j}\right)=0$ for all $j$.

Consider the set

$$
E(\gamma)=\left\{x \in \mathrm{S}_{\mu} ; \varlimsup_{r \searrow 0} \frac{\log \mu(\mathrm{B}(x, r))}{\log r}>\gamma\right\} .
$$

If $x \in E(\gamma)$, for all $\delta>0$, there exists $r \leq \delta$ such that $\mu(\mathrm{B}(x, r)) \leq r^{\gamma}$. Let $F$ be a subset of $E(\gamma)$. Set $F_{j}=F \cap E_{j}$.

For $\delta>0$, for all $j$, one can find a Besicovitch $\delta$-cover $\left\{\mathrm{B}_{j, k}\right\}$ of $F_{j}$ such that $\mu\left(\mathrm{B}_{j, k}\right) \leq r_{j, k}^{\gamma}$.

We have,

$$
\bar{\nu}_{\delta}\left(F_{j}\right) \leq \sum_{k} \nu\left(\mathrm{B}_{j, k}\right)=\sum_{k} \mu\left(\mathrm{B}_{j, k}\right)^{-t} \mu\left(\mathrm{B}_{j, k}\right)^{t} \nu\left(\mathrm{B}_{j, k}\right) \leq \sum_{k} \mu\left(\mathrm{B}_{j, k}\right)^{-t} r_{j, k}^{\gamma t} \nu\left(\mathrm{B}_{j, k}\right),
$$

which, together with the Besicovitch property, implies

$$
\bar{\nu}_{\delta}\left(F_{j}\right) \leq C_{B} \overline{\mathscr{P}}_{(\mu, \nu), \delta}^{(-t, 1), \gamma t}\left(E_{j}\right) .
$$

So

$$
\bar{\nu}\left(F_{j}\right) \leq C_{B} \overline{\mathscr{P}}_{(\mu, \nu)}^{(-t, 1), \gamma t}\left(E_{j}\right)=0 .
$$

This implies $\bar{\nu}(F)=0$, and $\nu^{\sharp}(E(\gamma))=0$. 
We conclude that

$$
\nu^{\sharp}\left(\left\{x \in \mathrm{S}_{\mu} ; \varlimsup_{r \searrow 0} \frac{\log \mu(\mathrm{B}(x, r))}{\log r}>-\varphi_{l}^{\prime}(0)\right\}\right)=0 .
$$

In the same way, one proves that

$$
\nu^{\sharp}\left(\left\{x \in \mathrm{S}_{\mu} ; \frac{\lim }{r \searrow 0} \frac{\log \mu(\mathrm{B}(x, r))}{\log r}<-\varphi_{r}^{\prime}(0)\right\}\right)=0 .
$$

Corollary 3.3. With the same notations and hypotheses as in Lemma 3.2, one has

$$
\operatorname{dim}_{H} X_{\mu}\left(-\varphi_{r}^{\prime}(0),-\varphi_{l}^{\prime}(0)\right) \geq \inf \left\{\frac{\lim }{r \searrow 0} \frac{\log \nu(\mathrm{B}(x, r))}{\log r} ; x \in X_{\mu}\left(-\varphi_{r}^{\prime}(0),-\varphi_{l}^{\prime}(0)\right)\right\}
$$

and

$\operatorname{dim}_{P} X_{\mu}\left(-\varphi_{r}^{\prime}(0),-\varphi_{l}^{\prime}(0)\right) \geq \inf \left\{\varlimsup_{r \searrow 0} \frac{\log \nu(\mathrm{B}(x, r))}{\log r} ; x \in X_{\mu}\left(-\varphi_{r}^{\prime}(0),-\varphi_{l}^{\prime}(0)\right)\right\}$.

Note that statements in Corollary 3.3 are weaker than what can be deduced from Lemma 3.2 and Lemma 3.1-b.

The previous lemmas contain the now classical results on multifractal analysis [8], [3], [10]. Indeed, let $\mu$ be a element of $\mathscr{F}^{*}$. Until the end of this section, we will write $b, \tau$, and $B$ instead of $b_{\mu, \mathrm{S}_{\mu}}, \tau_{\mu, \mathrm{S}_{\mu}}$, and $B_{\mu, \mathrm{S}_{\mu}}$. For $q \geq 0$, take $\nu(\mathrm{B})=\mu(\mathrm{B})^{q} r^{B(q)}$. Then the corresponding $\varphi$ of Lemma 3.2 is $B_{(\mu, \nu), \mathrm{S}_{\mu}}(t, 1)=$ $B(q+t)-B(q)$ and, for $x \in \bar{X}_{\mu}(\alpha)$, one has

$$
\varlimsup_{r \searrow 0} \frac{\log \nu(\mathrm{B}(x, r))}{\log r}=q \varlimsup_{r \searrow 0} \frac{\log \mu(\mathrm{B}(x, r))}{\log r}+B(q) \leq q \alpha+B(q) .
$$

So, by (3.2) of Lemma 3.1, one gets

$$
\operatorname{dim}_{P} \bar{X}_{\mu}(\alpha) \leq \inf _{q \geq 0} q \alpha+B(q)
$$

In the same way, we get

$$
\operatorname{dim}_{P} \underline{X}_{\mu}(\alpha) \leq \inf _{q \leq 0} q \alpha+B(q)
$$

If moreover we assume that $\mathscr{H}_{\mu}^{q, B(q)}\left(\mathrm{S}_{\mu}\right)>0$, we have $\nu^{\sharp}\left(\mathrm{S}_{\mu}\right)>0$, and therefore, by Lemma 3.2,

$$
\nu^{\sharp}\left(\left\{X_{\mu}\left(-B_{r}^{\prime}(q),-B_{l}^{\prime}(q)\right)\right\}\right)>0 .
$$

Therefore, by (3.3) of Lemma 3.1, we have

$$
\operatorname{dim}_{H}\left\{X_{\mu}\left(-B_{r}^{\prime}(q),-B_{l}^{\prime}(q)\right)\right\} \geq \begin{cases}-q B_{r}^{\prime}(q)+B(q) & \text { if } q \geq 0 \\ -q B_{l}^{\prime}(q)+B(q) & \text { if } q \leq 0\end{cases}
$$


Recall that the Legendre transform of a function $\chi$ is defined to be $\chi^{*}(\alpha)=$ $\inf _{q \in \mathbb{R}} q \alpha+\chi(q)$.

All this gives a new proof of the following theorem (see [2] in the totally disconnected case, [3] in general).

Theorem 3.4. If $B$ has a derivative at some point $q \in J_{B}$ and if $\mathscr{H}_{\mu}^{q, B(q)}\left(\mathrm{S}_{\mu}\right)>0$, then

$$
\operatorname{dim}_{H} X_{\mu}\left(-B^{\prime}(q)\right)=B^{*}\left(-B^{\prime}(q)\right) .
$$

The same statement holds with $\tau$ instead of $B$.

In [3] it is shown that if $B^{\prime}(q)$ exists and if $\operatorname{dim}_{H} X_{\mu}\left(-B^{\prime}(q)\right)=B^{*}\left(-B^{\prime}(q)\right)$, then $b(q)=B(q)$.

We now deal with the case when $b(q) \neq B(q)$. The following notation will prove convenient: for a real function $\psi$, we set

$$
\psi_{l}^{b}(q)=\varlimsup_{t \searrow 0} \frac{\psi(q-t)-\psi(q)}{-t} \quad \text { and } \quad \psi_{r}^{b}(q)=\varlimsup_{t \searrow 0} \frac{\psi(q+t)-\psi(q)}{t} .
$$

Lemma 3.5. Let $\mu$ and $\nu$ be elements of $\mathscr{F}^{*}$ and $\mathscr{F}$ respectively. Set $\varphi(t)=$ $b_{(\mu, \nu), \mathrm{S}_{\mu}}(t, 1)$ and assume that $\varphi(0)=0$ and $\nu^{\sharp}\left(\mathrm{S}_{\mu}\right)>0$. Then one has

$$
\nu^{\sharp}\left(\left\{x \in \mathrm{S}_{\mu} ; \frac{\lim }{r \searrow 0} \frac{\log \mu(\mathrm{B}(x, r))}{\log r}>-\varphi_{l}^{b}(0)\right\}\right)=0
$$

and

$$
\nu^{\sharp}\left(\left\{x \in \mathrm{S}_{\mu} ; \varlimsup_{r \searrow 0} \frac{\log \mu(\mathrm{B}(x, r))}{\log r}<-\varphi_{r}^{b}(0)\right\}\right)=0 .
$$

Proof. Take $\gamma>-\varphi_{l}^{b}(0)=\underline{\lim }_{t \searrow 0} \frac{\varphi(-t)}{t}$ and choose $t>0$ such that $\gamma t>\varphi(-t)$. We have $\mathscr{H}_{(\mu, \nu)}^{(-t, 1), \gamma t}\left(\mathrm{~S}_{\mu}\right)=0$.

Consider the set

$$
E=\left\{x \in \mathrm{S}_{\mu} ; \frac{\lim }{r \searrow 0} \frac{\log \mu(\mathrm{B}(x, r))}{\log r}>\gamma\right\} .
$$

For all $x \in E$, there exists $\delta>0$ such that, for all $r<\delta$, one has $\mu(\mathrm{B}(x, r))<r^{\gamma}$.

Set $E_{n}=\left\{x \in \mathrm{S}_{\mu} ; \forall r \leq 1 / n, \mu(\mathrm{B}(x, r))<r^{\gamma}\right\}$ and consider a subset $F$ of $E_{n}$. If $\left\{\mathrm{B}_{j}\right\}_{j}$ is any centered $\delta$-cover of $F$ with $\delta<1 / n$, one has

$$
\bar{\nu}_{\delta}(F) \leq \sum \nu\left(\mathrm{B}_{j}\right)=\sum \mu\left(\mathrm{B}_{j}\right)^{-t} \mu\left(\mathrm{B}_{j}\right)^{t} \nu\left(\mathrm{B}_{j}\right) \leq \sum \mu\left(\mathrm{B}_{j}\right)^{-t} r_{j}^{\gamma t} \nu\left(\mathrm{B}_{j}\right) .
$$

Therefore

Then we have

$$
\bar{\nu}_{\delta}(F) \leq \overline{\mathscr{H}}_{(\mu, \nu), \delta}^{(-t, 1), \gamma t}(F)
$$

$$
\bar{\nu}(F) \leq \overline{\mathscr{H}}_{(\mu, \nu)}^{(-t, 1), \gamma t}(F) \leq \mathscr{H}_{(\mu, \nu)}^{(-t, 1), \gamma t}\left(\mathrm{~S}_{\mu}\right)=0 .
$$

This implies $\nu^{\sharp}\left(E_{n}\right)=0$ and $\nu^{\sharp}(E)=0$. This proves the first assertion. The second one is proved in the same way. 
Proposition 3.6. Let $\mu$ be an element of $\mathcal{F}$. Suppose that, for some $q \in J_{b}$, $\mathscr{H}_{\mu}^{q, b(q)}\left(\mathrm{S}_{\mu}\right)>0$, and consider the set

$$
E=\left\{x \in \mathrm{S}_{\mu} ; \underline{\lim }_{r \searrow 0} \frac{\log \mu(\mathrm{B}(x, r))}{\log r} \leq-b_{l}^{b}(q) \text { and } \varlimsup_{r \searrow 0} \frac{\log \mu(\mathrm{B}(x, r))}{\log r} \geq-b_{r}^{b}(q)\right\} .
$$

Then we have

$$
\operatorname{dim}_{P} E \geq \begin{cases}b(q)-q b_{r}^{b}(q), & \text { if } q \geq 0 \\ b(q)-q b_{l}^{b}(q), & \text { if } q \leq 0\end{cases}
$$

In particular, if $b^{\prime}(q)$ exists one has

$\operatorname{dim}_{P}\left\{x \in \mathrm{S}_{\mu} ; \frac{\lim }{r \searrow 0} \frac{\log \mu(\mathrm{B}(x, r))}{\log r} \leq-b^{\prime}(q) \leq \varlimsup_{r \searrow 0} \frac{\log \mu(\mathrm{B}(x, r))}{\log r}\right\} \geq b(q)-q b^{\prime}(q)$.

Proof. This results from Lemma 3.5 and (3.4) of Lemma 3.1.

\section{An example}

Now, we can deal with the example given in [3] (Theorem 2.6). We take for $\mathbb{X}$ the space $\{0,1\}^{\mathbb{N}^{*}}$ endowed with the ultrametric which assigns diameter $2^{-n}$ to cylinders of order $n$.

We are given two numbers $p$ and $\tilde{p}$ such that $0<p<\tilde{p} \leq 1 / 2$, and a sequence of integers $1=t_{0}<t_{1}<\cdots<t_{n}<\cdots$ such that $\lim _{n \rightarrow \infty} t_{n} / t_{n+1}=0$.

We define a probability measure $\mu$ on $\{0,1\}^{\mathbb{N}^{*}}$ : the measure assigned to the cylinder $\left[\varepsilon_{1} \varepsilon_{2} \ldots \varepsilon_{n}\right]$ is

$$
\mu\left(\left[\varepsilon_{1} \varepsilon_{2} \ldots \varepsilon_{n}\right]\right)=\prod_{j=1}^{n} \varpi_{j}
$$

where

- if $t_{2 k-1} \leq j<t_{2 k}$ for some $k$, then $\varpi_{j}=p$ if $\varepsilon_{j}=0$, and $\varpi_{j}=1-p$ otherwise,

- if $t_{2 k} \leq j<t_{2 k+1}$ for some $k$, then $\varpi_{j}=\tilde{p}$ if $\varepsilon_{j}=0$, and $\varpi_{j}=1-\tilde{p}$ otherwise.

In fact, the measure considered in [3] is obtained by taking the image of $\mu$ under the natural binary coding of numbers in $[0,1]$ composed with the Gray code. The purpose of using the Gray code was to get a doubling measure on $[0,1]$.

For $q \in \mathbb{R}$, define

$$
\theta(q)=\log _{2}\left(p^{q}+(1-p)^{q}\right) \text { and } \tilde{\theta}(q)=\log _{2}\left(\tilde{p}^{q}+(1-\tilde{p})^{q}\right) .
$$

Then it follows from [3] that for $0<q<1$ we have

$$
b(q)=\theta(q)<\tilde{\theta}(q)=B(q),
$$

and, for $q<0$ or $q>1$,

$$
b(q)=\tilde{\theta}(q)<\theta(q)=B(q) .
$$


We wish to prove the following result:

Proposition 4.1. 1) For $\alpha \in\left(-\log _{2}(1-\tilde{p}),-\log _{2} \tilde{p}\right)$, we have

$$
\operatorname{dim}_{H} X_{\mu}(\alpha)=\inf _{q \in \mathbb{R}} b(q)+\alpha q
$$

2) For $\alpha \in\left(-\log _{2}(1-\tilde{p}),-\log _{2} \tilde{p}\right) \backslash\left(\left[-B_{r}^{\prime}(0),-B_{l}^{\prime}(0)\right] \cup\left[-B_{r}^{\prime}(1),-B_{l}^{\prime}(1)\right]\right)$, we have

$$
\operatorname{dim}_{P} X_{\mu}(\alpha)=\inf _{q \in \mathbb{R}} B(q)+\alpha q
$$

Proof. We consider the measure $\nu$ constructed as $\mu$ with parameters $r$ and $\tilde{r}$ instead of $p$ and $\tilde{p}$. We impose the condition

$$
r \log p+(1-r) \log (1-p)=\tilde{r} \log \tilde{p}+(1-\tilde{r}) \log (1-\tilde{p}) .
$$

As both $r$ and $\tilde{r}$ should belong to the interval $(0,1)$, we must have

$$
\log \frac{1-p}{1-\tilde{p}}<r \log \frac{1-p}{p}<\log \frac{1-p}{\tilde{p}}
$$

From Corollary 2.2, it is easy to compute $\varphi(x)=\tau_{(\mu, \nu), \mathrm{S}_{\mu}}$. We have

$$
\varphi(x)=\log _{2} \max \left\{\left(p^{x} r+(1-p)^{x}(1-r)\right),\left(\tilde{p}^{x} \tilde{r}+(1-\tilde{p})^{x}(1-\tilde{r})\right)\right\} .
$$

Condition (4.1) implies that $\varphi^{\prime}(0)$ exists. We set

$$
\alpha=-\varphi^{\prime}(0)=-r \log _{2} p-(1-r) \log _{2}(1-p)=r \log _{2} \frac{1-p}{p}-\log _{2}(1-p)
$$

It results from $(4.2)$ that $\alpha$ can take any value in the interval $\left(-\log _{2}(1-\tilde{p}),-\log _{2} \tilde{p}\right)$.

Moreover, the strong law of large numbers shows that we have

$$
\varliminf_{n \rightarrow \infty} \frac{\log _{2} \nu\left(\mathrm{B}\left(x, 2^{-n}\right)\right)}{-n}=\min \{\mathrm{h}(r), \mathrm{h}(\tilde{r})\}
$$

and

$$
\varlimsup_{n \rightarrow \infty} \frac{\log _{2} \nu\left(\mathrm{B}\left(x, 2^{-n}\right)\right)}{-n}=\max \{\mathrm{h}(r), \mathrm{h}(\tilde{r})\}
$$

for $\nu$-almost every $x$, where we set $\mathrm{h}(r)=-r \log _{2} r-(1-r) \log _{2}(1-r)$.

Then it results from Lemmas 3.2 and 3.1-b that

$$
\operatorname{dim}_{H} X_{\mu}(\alpha) \geq \min \{\mathrm{h}(r), \mathrm{h}(\tilde{r})\}
$$

and

$$
\operatorname{dim}_{P} X_{\mu}(\alpha) \geq \max \{\mathrm{h}(r), \mathrm{h}(\tilde{r})\},
$$

where $r, \tilde{r}$, and $\alpha$ are linked by (4.1) and (4.3). 
If $\alpha$ is defined by (4.3), we have

$$
\alpha=-\theta^{\prime}(q) \quad \text { if } \quad q=\frac{\log \frac{1-r}{r}}{\log \frac{1-p}{p}} \quad \text { and } \quad \alpha=-\tilde{\theta}^{\prime}(\tilde{q}) \quad \text { if } \quad \tilde{q}=\frac{\log \frac{1-\tilde{r}}{\tilde{r}}}{\log \frac{1-\tilde{p}}{\tilde{p}}} .
$$

Now fix $q$ and $\tilde{q}$ as above in (4.6). One can check that, for these values of $q$ and $\tilde{q}$, one has

$$
\theta(q)-q \theta^{\prime}(q)=\mathrm{h}(r) \text { and } \quad \tilde{\theta}(\tilde{q})-\tilde{q} \tilde{\theta}^{\prime}(\tilde{q})=\mathrm{h}(\tilde{r})
$$

In order to have $\theta(q)=b(q)$, we must have $0<q<1$, which means

$$
\log _{2} \frac{1}{p^{p}(1-p)^{1-p}}<\alpha<\log _{2} \frac{1}{\sqrt{p(1-p)}} .
$$

In order to have $\tilde{\theta}(\tilde{q})=b(\tilde{q})$, we must have $\tilde{q}<0$ or $\tilde{q}>1$, which means

$$
\alpha>\log _{2} \frac{1}{\sqrt{\tilde{p}(1-\tilde{p})}}
$$

or

$$
\alpha<\log _{2} \frac{1}{\tilde{p}^{\tilde{p}}(1-\tilde{p})^{1-\tilde{p}}}
$$

One can check that at least one of the conditions (4.8), (4.9) and (4.10) is fulfilled.

But for any $q$ such that $b^{\prime}(q)$ exists, we have (see [8] or [1]) that

$$
\operatorname{dim}_{H} X_{\mu}\left(-b^{\prime}(q)\right) \leq b(q)-q b^{\prime}(q) .
$$

The first assertion then results from (4.4), (4.7), and (4.11).

In order to have $\theta(q)=B(q)$, we must have $q<0$ or $q>1$, which means

$$
\alpha>\log _{2} \frac{1}{\sqrt{p(1-p)}}=-B_{l}^{\prime}(0) \quad \text { or } \quad \alpha<\log _{2} \frac{1}{p^{p}(1-p)^{1-p}}=-B_{r}^{\prime}(1) .
$$

In order to have $\tilde{\theta}(\tilde{q})=B(\tilde{q})$, we must have $0<\tilde{q}<1$, which means

$$
-B_{l}^{\prime}(1)=\log _{2} \frac{1}{\tilde{p}^{\tilde{p}}(1-\tilde{p})^{1-\tilde{p}}}<\alpha<\log _{2} \frac{1}{\sqrt{\tilde{p}(1-\tilde{p})}}=-B_{r}^{\prime}(0) .
$$

Then assertion (2) follows as before.

Remark 4.2. Proposition 4.1 also holds for the measure considered in [3]. Indeed, using the Gray code before projecting on $[0,1]$ yields doubling measures. 


\section{The vector case}

As in [10] one may consider expressions of the form $\exp -\langle q, \varkappa(\mathrm{B})\rangle$ instead of $\mu(\mathrm{B})^{q}$, where $\varkappa$ takes its values in the dual $\mathbb{E}^{\prime}$ of a separable Banach space $\mathbb{E}$ and $q \in \mathbb{E}$.

Let $\nu$ be an element of $\mathscr{F}$. For $E \subset \mathbb{X}, q \in \mathbb{E}, t \in \mathbb{R}$, and $\delta>0$, one sets

$$
\begin{aligned}
& \mathscr{P}_{\delta}^{q, t}(E)=\sup \left\{\sum r_{j}^{t} \mathrm{e}^{-\left\langle q, \varkappa\left(\mathrm{B}_{j}\right)\right\rangle} \nu\left(\mathrm{B}_{j}\right) ;\left\{\mathrm{B}_{j}\right\} \text { a } \delta \text {-packing of } E\right\}, \\
& \mathscr{P}^{q, t}(E)=\lim _{\delta \searrow 0} \overline{\mathscr{P}}_{\delta}^{q, t}(E), \\
& \mathscr{P}^{q, t}(E)=\inf \left\{\sum \overline{\mathscr{P}}^{q, t}\left(E_{j}\right) ; E \subset \bigcup E_{j}\right\},
\end{aligned}
$$

and

$$
\begin{aligned}
& \overline{\mathscr{H}}_{\delta}^{q, t}(E)=\inf \left\{\sum r_{j}^{t} \mathrm{e}^{-\left\langle q, \varkappa\left(\mathrm{B}_{j}\right)\right\rangle} \nu\left(\mathrm{B}_{j}\right) ;\left\{\mathrm{B}_{j}\right\} \text { a centered } \delta \text {-cover of } E\right\}, \\
& \overline{\mathscr{H}}^{q, t}(E)=\lim _{\delta \searrow 0} \overline{\mathscr{H}}_{\delta}^{q, t}(E), \\
& \mathscr{H}^{q, t}(E)=\sup \left\{\overline{\mathscr{H}}^{q, t}(F) ; F \subset E\right\},
\end{aligned}
$$

For a function $\chi$ from $\mathbb{E}$ to $\mathbb{R}$, and for $v \in \mathbb{E}$ of norm 1 , one defines

$$
\partial_{v} \chi(0)=\lim _{t \searrow 0} \frac{\chi(t v)-\chi(0)}{t} \text { and } \partial_{v}^{*} \chi(0)=\varlimsup_{t \searrow 0}-\frac{\chi(t v)-\chi(0)}{t} .
$$

With these notations we have the following analogues of Lemmas 3.2 and 3.5:

Lemma 5.1. Let $\varphi(q)$ be one of the following functions:

$$
\inf \left\{t ; \overline{\mathscr{P}}^{q, t}(\mathbb{X})=0\right\} \quad \text { or } \quad \inf \left\{t ; \mathscr{P}^{q, t}(\mathbb{X})=0\right\} \text {. }
$$

Assume that $\varphi(0)=0$ and that $\partial_{v} \varphi(0)$ at 0 is a lower semi-continuous function of $v$. Then one has

$$
\nu^{\sharp}\left\{x ; \frac{\lim }{r \searrow 0} \frac{\langle v, \varkappa(\mathrm{B}(x, r))}{-\ln r}<-\partial_{v} \varphi(0) \text { for some } v \in \mathbb{E}\right\}=0 .
$$

Lemma 5.2. Set $\varphi(q)=\inf \left\{t ; \mathscr{H}^{q, t}(\mathbb{X})=0\right\}$ and assume that $\varphi(0)=0$ and that $\partial_{v}^{*} \chi(0)$ is a lower semi-continuous function of $v$. Then one has

$$
\nu^{\sharp}\left\{x ; \varlimsup_{r \searrow 0} \frac{\langle v, \varkappa(\mathrm{B}(x, r))}{-\ln r}<-\partial_{v}^{*} \varphi(0) \text { for some } v \in \mathbb{E}\right\}=0 .
$$

The proofs follow the same lines as those above and as the proofs in [10]. As a corollary we get the following result (with the notations of [10]):

Theorem 5.3. Let $B(q)=\inf \left\{t \in \mathbb{R} ; \mathscr{H}_{\varkappa}^{q, t}(\mathbb{X})=0\right\}$. Assume that, at some point $q$, the function $B$ is differentiable with derivative $B^{\prime}(q)$ and that $\mathscr{H}_{\varkappa}^{q, B(q)}(\mathbb{X})>0$. Then one has

$$
\operatorname{dim}_{H}\left\{x ; \forall v \in \mathbb{E}, \lim _{r \searrow 0} \frac{\langle v, \varkappa(\mathrm{B}(x, r))\rangle}{\log r}=-B^{\prime}(q) v\right\}=B(q)-B^{\prime}(q) q .
$$




\section{References}

[1] Barral, J., Fan, A. H. And Peyrière, J.: Mesures engendrées par multiplications. In Quelques interactions entre analyse, probabilités et fractals, 57-189. Panor. Syntheses 32, Soc. Math. France, Paris, 2010.

[2] Ben Nasr, F.: Analyse multifractale de mesures. C. R. Acad. Sci. Paris Sér. I Math. 319 (1994), 807-810.

[3] Ben Nasr, F., Bhouri, I. and Heurteaux, Y.: The validity of the multifractal formalism: results and examples. Adv. Math. 165 (2002), 264-284.

[4] Billingsley, P.: Ergodic theory and information. John Wiley, New York-LondonSydney, 1965.

[5] Frisch, U. AND PARISI, U.: On the singularity structure of fully developed turbulence (appendix to "Fully developed turbulence and intermittency", by U. Frisch). In Proceedings of the International School of Physics "Enrico Fermi", 84-88. NorthHolland, 1985.

[6] Halsey, T. C., Jensen, M. H., Kadanoff, L. P., Procaccia, I. and Shraiman, B. I.: Fractal measures and their singularities: the characterisation of strange sets. Phys. Rev. A (3) 33 (1986), 1141-1151.

[7] Hentschel, H. G. and Procaccia, I.: The infinite number of generalized dimensions of fractals and strange attractors. Phys. D 8 (1983), 435-444.

[8] Olsen, L.: A multifractal formalism. Adv. Math. 116 (1995), 82-196.

[9] Pesin, Y.: Dimension theory in dynamical systems. Contemporary views and applications, Chicago Lectures in Mathematics, University of Chicago Press, Chicago, IL, 1997.

[10] Peyrière, J.: A vectorial multifractal formalism. In Fractal geometry and applications: a jubilee of Benoît Mandelbrot, Part 2, 217-230. Proc. Sympos. Pure Math. 72, Part 2, Amer. Math. Soc., Providence, RI, 2004.

[11] Saint Raymond, X. And Tricot, C.: Packing regularity of sets in $n$-space. Math. Proc. Cambridge Philos. Soc. 103 (1988), 133-145.

[12] Tricot, C.: Two definitions of fractional dimension. Math. Proc. Cambridge Philos. Soc. 91 (1982), 57-74.

Received May 14, 2011.

F. Ben NasR: Département de Mathématiques, Faculté des Sciences de Monastir, Monastir 5000, Tunisie.

E-mail: fathi_bennasr@yahoo.fr

J. Peyrière: Université Paris-Sud, Mathématique bât. 425, CNRS UMR 8628, 91405 Orsay Cedex, France; and School of Mathematics and Systems Science, Beihang University, Beijing 100191, P. R. China.

E-mail: jacques.peyriere@math.u-psud.fr

J. Peyrière gratefully acknowledges the support of Project 111, P. R. China. 